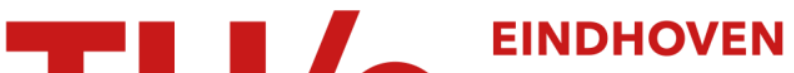

\section{Radiating plasma species density distribution in EUV-induced plasma in argon: a spatiotemporal experimental study}

\section{Citation for published version (APA):}

Van Der Horst, R. M., Beckers, J., Osorio, E. A., van de Ven, T. H. M., \& Banine, V. Y. (2015). Radiating plasma species density distribution in EUV-induced plasma in argon: a spatiotemporal experimental study. Plasma Sources Science and Technology, 24(6), 1-10. [065016]. https://doi.org/10.1088/0963-0252/24/6/065016

\section{Document license: \\ TAVERNE}

DOI:

10.1088/0963-0252/24/6/065016

Document status and date:

Published: 01/12/2015

\section{Document Version:}

Publisher's PDF, also known as Version of Record (includes final page, issue and volume numbers)

\section{Please check the document version of this publication:}

- A submitted manuscript is the version of the article upon submission and before peer-review. There can be important differences between the submitted version and the official published version of record. People interested in the research are advised to contact the author for the final version of the publication, or visit the $\mathrm{DOI}$ to the publisher's website.

- The final author version and the galley proof are versions of the publication after peer review.

- The final published version features the final layout of the paper including the volume, issue and page numbers.

Link to publication

\section{General rights}

Copyright and moral rights for the publications made accessible in the public portal are retained by the authors and/or other copyright owners and it is a condition of accessing publications that users recognise and abide by the legal requirements associated with these rights.

- Users may download and print one copy of any publication from the public portal for the purpose of private study or research.

- You may not further distribute the material or use it for any profit-making activity or commercial gain

- You may freely distribute the URL identifying the publication in the public portal.

If the publication is distributed under the terms of Article 25fa of the Dutch Copyright Act, indicated by the "Taverne" license above, please follow below link for the End User Agreement:

www.tue.nl/taverne

Take down policy

If you believe that this document breaches copyright please contact us at:

openaccess@tue.nl

providing details and we will investigate your claim. 
Radiating plasma species density distribution in EUV-induced plasma in argon: a spatiotemporal experimental study

This content has been downloaded from IOPscience. Please scroll down to see the full text. 2015 Plasma Sources Sci. Technol. 24065016

(http://iopscience.iop.org/0963-0252/24/6/065016)

View the table of contents for this issue, or go to the journal homepage for more

Download details:

IP Address: 131.155.151.137

This content was downloaded on 08/12/2015 at 10:00

Please note that terms and conditions apply. 


\title{
Radiating plasma species density distribution in EUV-induced plasma in argon: a spatiotemporal experimental study
}

\author{
R M van der Horst ${ }^{1}$, J Beckers ${ }^{1}$, E A Osorio ${ }^{2}$, T H M van de Ven $^{1}$ and \\ V Y Banine ${ }^{1,2}$ \\ ${ }^{1}$ Department of Applied Physics, Eindhoven University of Technology, PO Box 513, 5600MB \\ Eindhoven, The Netherlands \\ 2 ASML The Netherlands B V, PO Box 324, 5500AH Veldhoven, The Netherlands \\ E-mail: r.m.v.d.horst@tue.nl
}

Received 25 August 2015, revised 1 October 2015

Accepted for publication 22 October 2015

Published 17 November 2015

\begin{abstract}
In this contribution we experimentally study temporally and spatially resolved radiating plasma species density distribution in plasma induced by irradiating a low pressure argon gas with high energy photons with a wavelength of $13.5 \mathrm{~nm}$, i.e. extreme ultraviolet (EUV). This is done by recording the optical emission spatially and temporally resolved by an iCCD camera as a function of the argon gas pressure. Our experimental results show that the emission intensity, i.e. density of radiating plasma species, depends quadratically on the gas pressure. The linear term is due to photoionization and simultaneous excitation by EUV photons, the quadratic term due to electron impact excitation by electrons generated by photoionization. The decay of radiating plasma species can be divided into two phases. At time scales shorter than $10 \mu$ s (first phase), the decay is governed by radiative decay of radiating plasma species. At longer time scales (second phase, $>10 \mu \mathrm{s}$ ), the decay is dominated by diffusion and subsequent de-excitation at the wall. The experimental decay and expansion during this phase corresponds well with a simplified diffusion model. In order to gain more insight in this exotic type of plasma, we compare the electron density from previous measurements with the results obtained here.
\end{abstract}

Keywords: optical emission, EUV, imaging

(Some figures may appear in colour only in the online journal)

\section{Introduction}

Although plasmas are studied in various fields of research, plasma induced due to photoionization of low pressure gas by high energy photons with an energy of $92 \mathrm{eV}$, i.e. extreme ultraviolet (EUV) radiation, is scarcely investigated. These type of plasmas are unique, since they are generated without the need of a discharge. EUV-induced plasmas occurs for instance in space, where it is a key process in the generation of various types of astrophysical plasmas close to strongly radiating objects [1-4]. Furthermore, EUV-induced plasma occurs in EUV lithography tools, which is the context of the research presented here.
The aforementioned semiconductor industry is continuously striving to increase the computational power and memory capacity of computer chips by reducing the size of the components of integrated circuits. This miniaturization is currently achieved by reducing the wavelength from UV $(192 \mathrm{~nm})$ to EUV at $13.5 \mathrm{~nm}$ [5], which corresponds to a photon energy of $92 \mathrm{eV}$. These high energy photons are absorbed by the background gas in the lithography tool, which leads to a plasma due to photoionization. This EUV- induced plasma is related to the lifetime of the optics in lithography tools, hence, a fundamental knowledge of EUV-induced plasma is essential to understand its long term impact on the optics. 


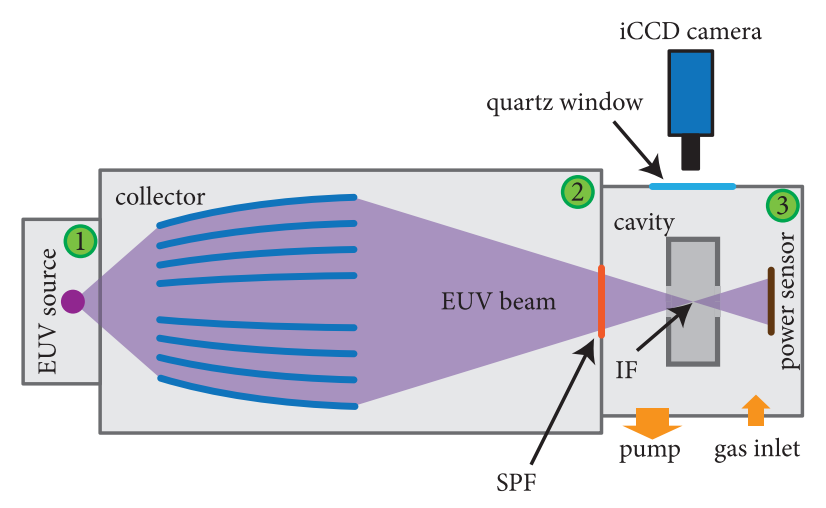

Figure 1. Schematic overview of the experimental set-up. A xenon source generates EUV radiation, which is collected by the collector and focused in the intermediate focus (IF). A mesh cavity is placed around IF. The plasma emission is recorded by an iCCD camera.

The measurement vessel is a CF160 cross piece, which has an inner diameter of about $160 \mathrm{~mm}$. This figure is adapted from [10].

A few years ago, Van der Velden [6, 7] studied EUVinduced plasma in argon using simulations. Furthermore, the authors attempted to measure the electron density using Langmuir probes, however, they concluded that these were not feasible in EUV- induced plasma [7]. Later, Dolgov et al [8] proposed a set-up to study the influence of EUV-induced plasma on the optical elements in lithography tools. Recently, we were the first to report non-invasive measurements of the electron density in EUV-induced plasmas in argon and hydrogen using a technique called microwave cavity resonance spectroscopy [9-12]. So far, only the evolution of the electron density was studied. During these experiments, it was clearly observed by eye that the EUV-induced plasma in argon emitted visible radiation. This contribution is devoted to in depth experimentally investigate the spatially and temporally resolved emission by a pulsed EUV-induced plasma in argon using a gated iCCD camera. Argon was used since it is one of the gases used in lithography tools and the emission intensity of an EUV-induced plasma in argon is significantly larger than in for instance hydrogen. The observed phenomena have been investigated as a function of argon pressure. Furthermore, the expansion of the radiating plasma species density distribution is compared to the expansion of the electron density distribution under the same conditions [12]. Finally, the influence of the cavity on the expansion of the radiating plasma species density distribution is assessed.

\section{Experimental set-up and method}

The experimental set-up consists of three chambers: the source chamber, the collector chamber and the measurement chamber (see figure 1). The xenon-based discharge produced EUV source, which is extensively described in [13, 14], is located in the source chamber; it is operated at a repetition rate of $500 \mathrm{~Hz}$, the duration of one EUV-pulse is $100 \mathrm{~ns}$ and the EUV pulse energy is about $120 \mu \mathrm{J}$ at IF. The generated EUV radiation is collected and focused in the intermediate focus (IF) by the collector, which is a set of rotationally symmetric grazing incidence mirrors located in the collector chamber.
In order to obtain high vacuum in the measurement chamber, the collector and measurement chamber are differentially pumped with an aperture of $4 \mathrm{~mm}$ in diameter; the base pressure in the measurement chamber is $10^{-4} \mathrm{~Pa}$, while the pressure in the collector chamber is $0.1 \mathrm{~Pa}$ when the source is in operation. A spectral purity filter (SPF), which transmits between $10-20 \mathrm{~nm}$, is placed in front of the aperture in the beam path. The EUV pulse energy is measured with an accuracy of 5\% using a power sensor as extensively discussed in [10]. A cavity is positioned around IF to mimic the conditions of previous electron density experiments [10].

Previously, the electron density is measured in similar plasma conditions and experimental set-up using microwave cavity resonance spectroscopy (MCRS) [10]. For the imaging experiments presented here, the cavity used for the MCRS measurements is slightly adapted in two ways, since the cavity for the MCRS measurements was made of solid aluminium. The cylindrical side-wall of the cavity was replaced by a stainless steel mesh with a thread thickness of $0.35 \mathrm{~mm}$, a mesh size of $1.08 \mathrm{~mm}$ and a transmission of $57 \%$. First, the mesh size is in the same order of magnitude as the Debye length in the decaying plasma, which means that the mesh can be seen as a solid wall for the plasma. It should be noted, however, that this is not valid for the neutral particles, which will (partially) penetrate through the mesh wall. Second, adaptation is that the antennas are not installed in the cavity for the imaging experiments.

An Andor 534 iCCD camera was used to image the optical emission of EUV-induced plasma spatially and temporally resolved. A CoastalOpt $105 \mathrm{~mm}$ UV-VIS f/4.5 apochromatic, i.e. corrected for chromatic and spherical aberrations, lens with a spectral range from $250-650 \mathrm{~nm}$ was mounted on the iCCD camera. By using the intensifier in front of the CCD camera, images with a nanosecond shutter time could be taken.

The camera is triggered with the trigger signal from the source. Since the trigger signal arrives at the camera after the EUV pulse, the start of the EUV plasma is studied by imaging the subsequent EUV pulse. This results in a lower time resolution at the start of the EUV pulse, since the jitter of the repetition time of the source is about 140 ns. The delay in the cables and different devices is taken into account, and in all presented images $t=0$ corresponds to the maximum of the EUV pulse. The camera software is used to set the delay between the trigger input and opening of the gate (gate delay), the time the gate is opened (gate width), the gain and the exposure time of the CCD. The used gate width depends on the emission intensity. By increasing this exposure time, the number of accumulated discharges increases. Depending on the emission intensity, 30 000-180 000 discharges are accumulated for constructing one image. Before imaging the EUV-induced plasma, the dark current is measured by closing the shutter of the iCCD camera and the background emission is measured by closing the shutter of the EUV beam. Afterwards the images are corrected for the background emission and dark current. Since all images are taken with different gate widths, gains and exposure times, the intensity of the images is corrected for these parameters. 

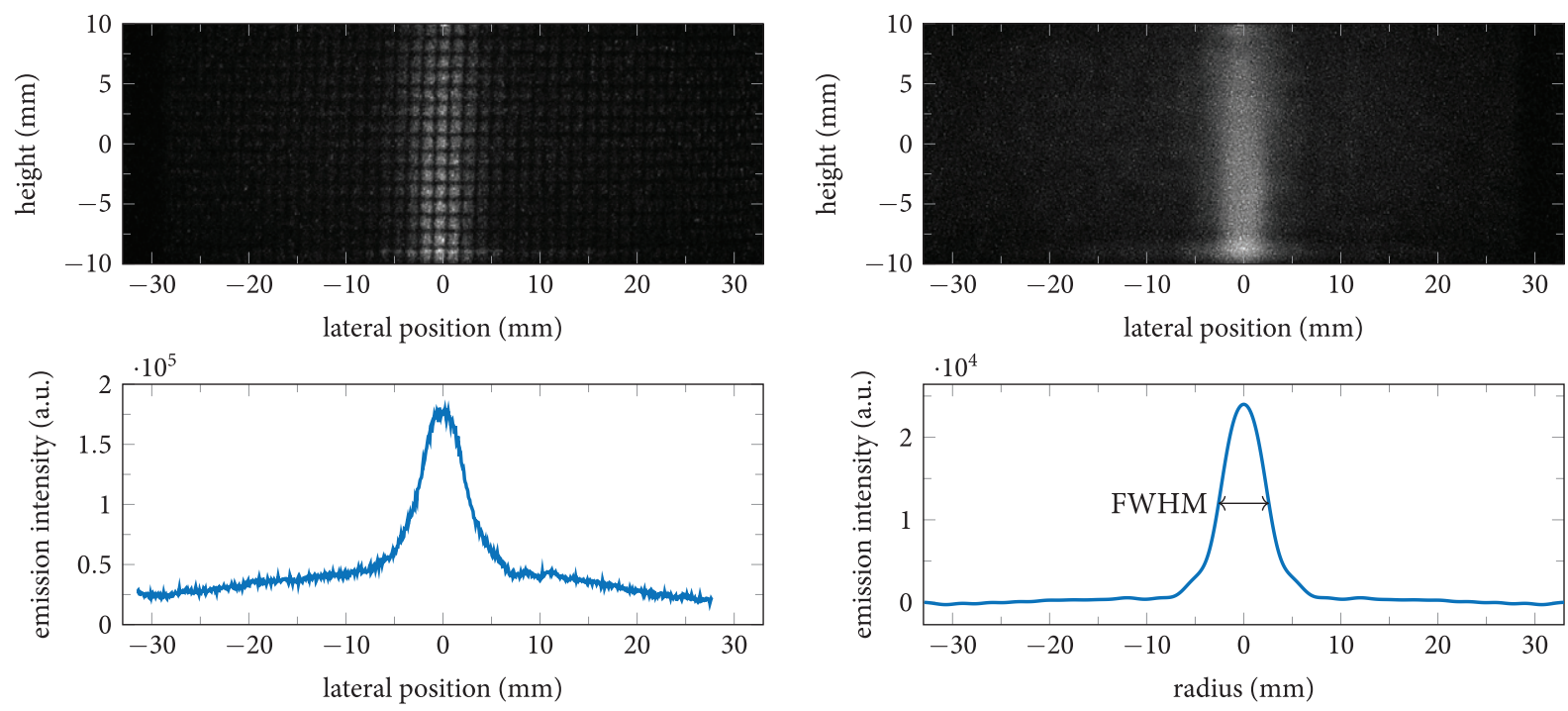

Figure 2. Example of the extraction of a radial profile from $2 \mathrm{D}$ emission measurements (number 5 in figure 5). On the top left is the $2 \mathrm{D}$ emission profile with grid and on the top right without grid. The bottom left graph shows the average emission intensity over the height of the cavity. The bottom right graph shows the Abel inversion and indicates the FWHM.

A spectrometer is used to study the spectrally resolved optical emission. An optical fibre is inserted in the measurement vessel at a radial distance of about $2 \mathrm{~cm}$ from the centre of the EUV beam. The fibre is connected to an Ocean Optics HR2000 (200-600 nm) via an optical feedtrough.

\subsection{Data post processing}

To obtain adequate information from the images, post-processing is executed as follows:

(i) The grid of the cavity, which is visible in the raw images (see figure 2 top left), is removed. The Fourier transform of the image is calculated and the frequency components due to the grid are removed. Afterwards the inverse Fourier transform is taken to retrieve the image without the grid (see figure 2 top right). This method would also remove the spatial variations in the plasma emission with the same spatial frequency as the mesh. However, these spatial variation are unlikely to occur in the plasma.

(ii) Only to determine the temporal evolution of the total plasma emission, the measured intensity is summed over the area of the cavity.

(iii) To study the expansion of the plasma, the radial distribution of the plasma emission is needed. To obtain this distribution, the lateral emission distribution needs to be determined. This is achieved by averaging the emission intensity over the height of the cavity for every radial position. Since there are some reflections visible on the walls of the cavity, the area within $1.5 \mathrm{~mm}$ from the walls is not included. Finally, the lateral position of the maximum of the lateral distribution is defined as $y=0$, i.e. the lateral distribution is centred around its maximum (see figure 2 bottom left).

(iv) Next, Abel inversion is performed according to the procedure described in [15] to obtain the radial profile (see figure 2 bottom right). (v) Finally, the full width at half maximum (FWHM) of the radial profiles obtained from the Abel inversion is determined (see figure 2 bottom right).

The imaging experiments are performed without the cavity as well, for which the analysis procedure described above is slightly adapted. In this case, the grid is not visible in the images and, hence, grid removal procedure is not applied. Since the spatially resolved plasma emission in these images shows no variation with the same frequency as the grid of the cavity, it is concluded that the removal of the grid as discussed before does not influence the spatially resolved emission intensity. To extract the lateral profile, the same area of the image is used as for the experiment with the cavity. However, due to the limited field of view of the camera, the lateral distribution is not always fully acquired, but the edges are missing. To account for this, the lateral distribution is linearly extrapolated until an intensity of zero. The radius $R$ in the Abel inversion is changed to the radius of the vacuum vessel, which is about $80 \mathrm{~mm}$.

\section{Temporal evolution of the total integrated emission intensity}

In this section, the temporal evolution of the total emission intensity is studied. First, in order to determine the source of the emitted light, a time-integrated spectrum of the plasma radiation obtained with an Ocean Optics HR2000 (200-600 nm) spectrometer is recorded (figure 3 ). The spectrum shows emission lines from both radiating argon states and radiating argon ion states, while it shows no significant continuum radiation. This means that the total emission recorded by the iCCD camera originates from both radiating exited argon atoms and ions.

The temporal evolution of the total emission intensity of a pulsed EUV-induced plasma in argon at a pressure of $5 \mathrm{~Pa}$ is plotted in figure 4 . The horizontal error bars represent the 


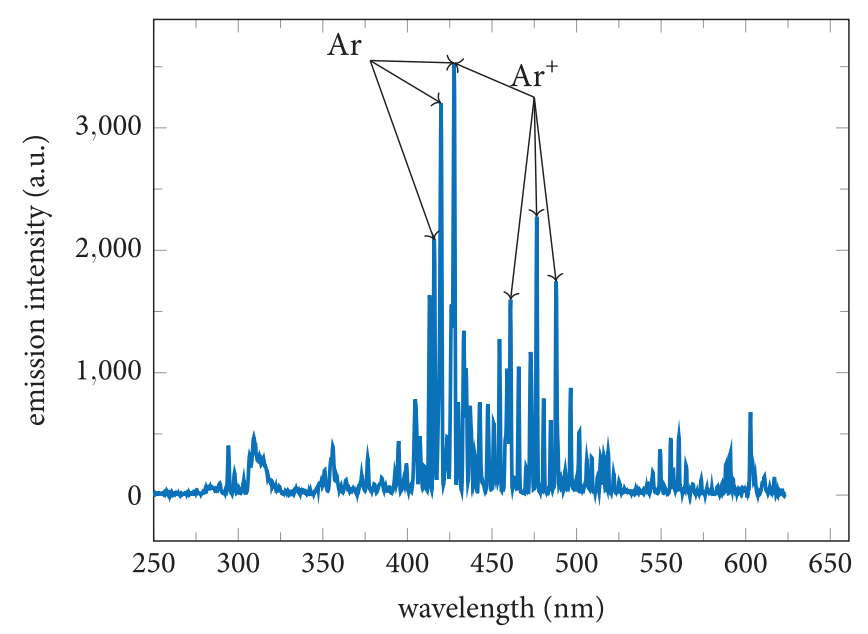

Figure 3. Time-integrated spectrum of an argon EUV-induced plasma at a pressure of $10 \mathrm{~Pa}$. The emission lines originate from both radiating excited argon atoms and ions, a few of them are indicated in the graph.

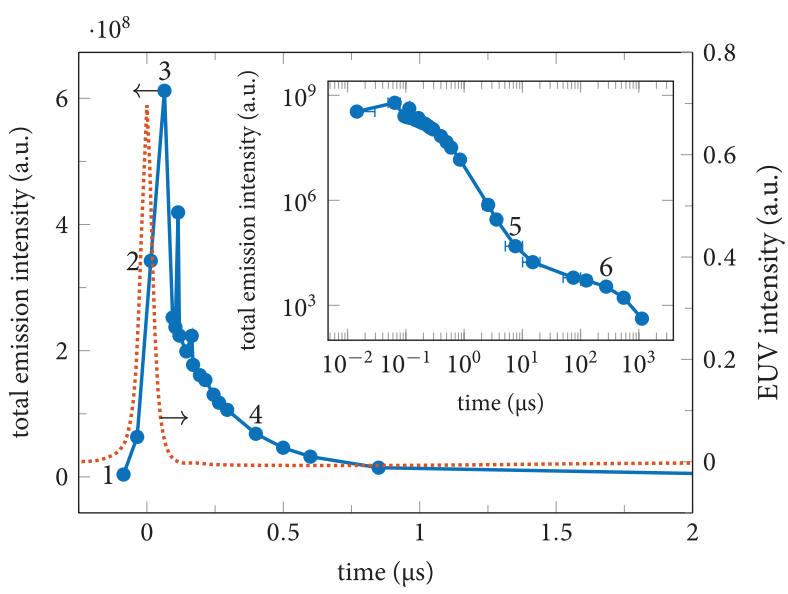

Figure 4. Total emission intensity of an EUV-induced plasma in argon at a pressure of $5 \mathrm{~Pa}$. The arbitrary intensity scale of the inset is similar as the scale of the main graphs. The numbers correspond to the images in figure 5 and the horizontal error bars represent the gate width of the iCCD camera.

gate width of the iCCD camera. Figure 4 shows that the emission intensity starts to increase at the moment the EUV pulse starts. At the end of the EUV pulse, the maximum emission intensity is reached. The decay can be divided in two phases: a fast decay before $10 \mu$ s and a slow decay after $10 \mu \mathrm{s}$. In the first phase, the total emission intensity rapidly decreases 4 orders of magnitude in less than $10 \mu$ s. Three processes can be responsible for the decrease of the total emission intensity: wall de-excitation, quenching and radiative decay. The first two mechanisms seem to be unlikely. During this rapid decrease, the radiating plasma species density distribution has not yet expanded to the wall by diffusion (see figure 5), which means that wall de-excitation does not govern this decrease. Furthermore, the typical quenching time is relatively long at a pressure of $5 \mathrm{~Pa}(0.3 \mathrm{~s})$ [16]. Hence, the rapid decrease is governed by the natural decay rate of the radiating plasma species, i.e. radiative decay. The typical natural lifetimes of the most intense emission lines of $\mathrm{Ar}$ and $\mathrm{Ar}^{+}$are less than $2 \mu \mathrm{s}$

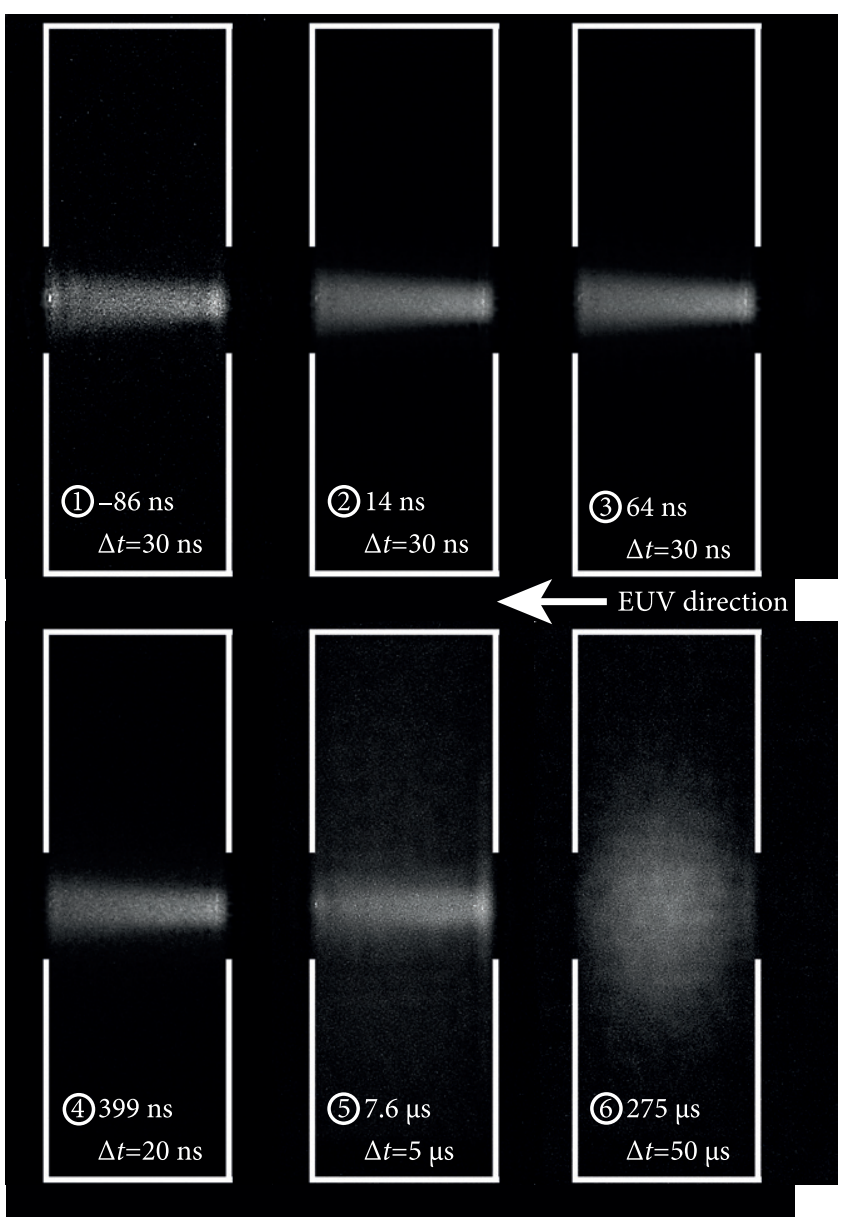

Figure 5. Images at various moments in time as indicated by the numbers in figure 4 . The time $\Delta t$ is the set gate width of the iCCD camera of the particular images. The EUV beam is directed through the centre of the cavity (the walls of the cavity are indicated by the white bars) from right to left. The intensities of the images are individually normalized.

(table 1), which is less than the observed time scale of about $10 \mu \mathrm{s}$. Other, less intense, lines of $\mathrm{Ar}$ and $\mathrm{Ar}^{+}$have a longer natural lifetime and cause the emission at much lower intensities in the second phase (table 2).

On long time scales (up to $1 \mathrm{~ms}$ after the EUV pulse), the plasma remains emitting visible light (see figure 4). Since the electron temperature is around room temperature on these time scales [18], electron impact excitation cannot generate new radiating plasma species. Hence, the emission must originate from long living radiating plasma species.

The normalized spatial emission profile is shown in figure 5 at various moments in time, in which the numbers in the images (1-6) correspond to the numbers in figure 4, while $\Delta t$ is the set gate width of the iCCD camera of the particular images. The EUV beam is directed through the centre of the cavity (the walls of the cavity are represented by the white bars) from right to left as indicated by the arrow. The following can be observed and speculated from the images. First, the images indicate that the radiating plasma species density distribution is initially confined to the EUV beam volume, i.e. the radial FWHM of the emission intensity profile is about equal to the FWHM of the EUV-beam, which is determined 
Table 1. The wavelength and natural lifetime of the most intense argon and argon ion lines [17].

\begin{tabular}{llcr}
\hline Species & $\begin{array}{l}\text { Wavelength } \\
(\mathrm{nm})\end{array}$ & $\begin{array}{l}\text { Natural } \\
\text { lifetime (ns) }\end{array}$ & $\begin{array}{l}\text { Intensity } \\
\text { (a.u.) }\end{array}$ \\
\hline $\mathrm{Ar}$ & 415.9 & 714 & 400 \\
$\mathrm{Ar}$ & 420.1 & 1034 & 400 \\
$\mathrm{Ar}$ & 427.2 & 1255 & 150 \\
$\mathrm{Ar}$ & 433.4 & 1761 & 100 \\
$\mathrm{Ar}^{+}$ & 413.2 & 12 & 891 \\
$\mathrm{Ar}^{+}$ & 427.8 & 13 & 1995 \\
$\mathrm{Ar}^{+}$ & 459.0 & 15 & 25704 \\
$\mathrm{Ar}^{+}$ & 472.7 & 17 & 23443 \\
$\mathrm{Ar}^{+}$ & 476.5 & 16 & 2344 \\
\hline
\end{tabular}

Table 2. The wavelength and natural lifetime of long living argon and argon ion lines [17].

\begin{tabular}{llcl}
\hline Species & $\begin{array}{l}\text { Wavelength } \\
(\mathrm{nm})\end{array}$ & $\begin{array}{l}\text { Natural } \\
\text { lifetime }(\mu \mathrm{s})\end{array}$ & $\begin{array}{l}\text { Intensity } \\
(\text { a.u. })\end{array}$ \\
\hline $\mathrm{Ar}$ & 436.4 & 83 & \\
$\mathrm{Ar}$ & 442.4 & 137 & \\
$\mathrm{Ar}$ & 458.9 & 161 & \\
$\mathrm{Ar}$ & 519.3 & 83 & 1 \\
$\mathrm{Ar}^{+}$ & 405.8 & 50 & \\
$\mathrm{Ar}^{+}$ & 406.1 & 313 & 2 \\
$\mathrm{Ar}^{+}$ & 414.7 & 56 & 0.5 \\
$\mathrm{Ar}^{+}$ & 521.5 & 63 & \\
\hline
\end{tabular}

Note: The relative intensity is missing from the NIST database for some of these lines.

from the discolouration of EUV sensitive foil $(4 \mathrm{~mm})$. This means that the radiating plasma species are mainly created along the EUV beam. Second, the waist of the initial emission intensity profile is not in the centre of the cavity, but located near the right wall. Since the maximum speed of the gas flow in our pumping system is $100 \mathrm{~m} \mathrm{~s}^{-1}$, gas flow can only move the radiating plasma species $\sim 0.1 \mathrm{~mm}$ before the excited state decays $(\sim 1 \mu \mathrm{s}$ [17]). Hence, the intermediate focus of the EUV beam is not located in the centre of the cavity. Third, the radiating plasma species density distribution expands towards the walls of the cavity in tens of microseconds, which is studied in detail in the next section.

During the experiments it was observed by eye that the EUV-induced plasma appears to be visually confined in space, which was also reported by Banine et al [19]. This can be explained as follows. As mentioned before, the emission intensity decreases rapidly after the EUV pulse $(<10 \mu \mathrm{s})$, while the radiating plasma species density distribution hardly expands during this rapid decrease. Hence, the emission intensity from this initial phase is the main contributor to the timeintegrated visible emission. This means that the observed emission observed by eye, which is time-integrated over multiple pulses since the eye can only observe at roughly 10 $\mathrm{Hz}[20]$, originates from the initial phase where the plasma emission has not yet expanded. Although the radiating volume expands eventually (see figure 5), this contribution to the total light emission is negligible.

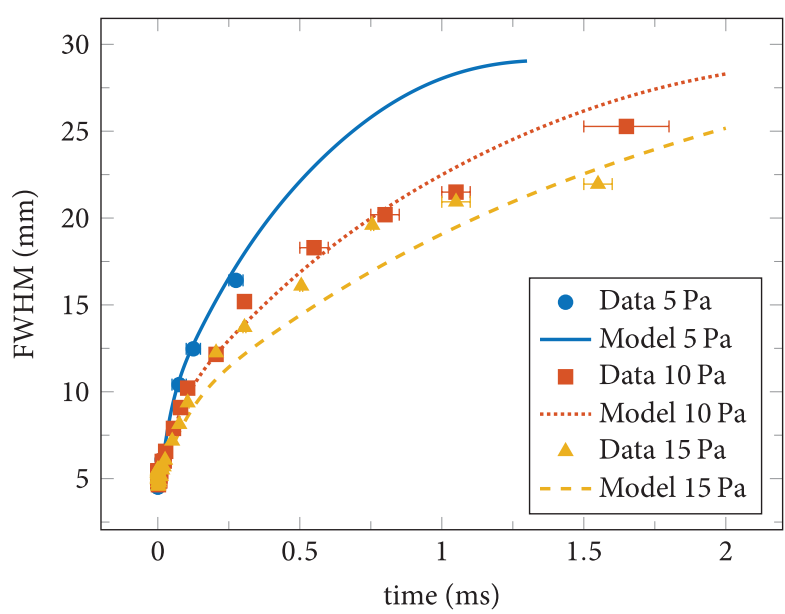

Figure 6. FWHM of the radial emission profile as a function of time for various argon pressures. The horizontal error bars represent the gate width of the iCCD camera. Both experimental results and the results from the diffusion model are shown.

Summarizing, the plasma starts emitting at the start of the EUV pulse. At the end of the EUV pulse, the emission intensity decays. Due to the initial rapid decay $(<10 \mu \mathrm{s})$ in which the radiating plasma species density distribution does not expand due to the inertia of the atoms and ions, the visible emission appears to be confined to the EUV beam by eye.

\section{The influence of the pressure on the expansion of the radiating plasma species density distribution}

In this section a study of the influence of the pressure on the expansion of the radiating plasma species density distribution is presented. Furthermore, the experimental results will be compared to a simplified neutral diffusion model, since it will appear later in this section that it is mainly the neutral species that account for the radiation on longer time scales.

To study this expansion, the radial emission profile is extracted from the images in figure 5. The procedure of this extraction is discussed in detail in section 2 .

The determined FWHM of the radial emission profile is plotted as a function of time for various pressures in figure 6 . The data shows that the expansion is slower at elevated pressures. This is an indication that the expansion is most likely governed by (neutral) diffusion, which scales with $D \propto p^{-1}$. To verify that diffusion dominates the expansion, the expansion is modelled with a simplified 2-dimensional diffusion model which solving the diffusion equation:

$$
\frac{\partial f(\vec{r}, t)}{\partial t}=D \nabla^{2} f(\vec{r}, t),
$$

where $D$ is the diffusion coefficient. As will be shown in section 7, the emission is dominated by radiating argon atoms on the investigated time scales $(>10 \mu \mathrm{s})$, which is why the diffusion coefficient of neutral argon in its parent gas $\left(0.72 \mathrm{~Pa} \mathrm{~m}^{-1} \mathrm{~s}^{-1}[16]\right)$ is used. Since the cavity is axisymmetric, it can be represented with a 2-dimensional (radius $r$ and height $z$ ) cylindrical geometry (see figure 7). The total computational grid has a radius of $33 \mathrm{~mm}$ and a height of $80 \mathrm{~mm}$. A Neumann 


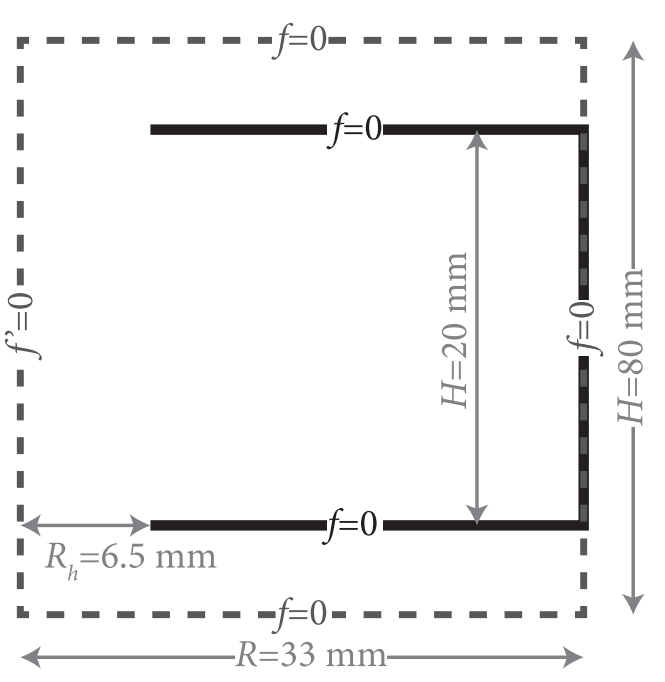

Figure 7. Schematic overview of the geometry and boundary conditions of the diffusion model. The left boundary is the axis of symmetry $(r=0)$.

boundary condition was applied on the $r=0$ axis and Dirichlet boundary conditions on the other boundaries of the grid. On the walls of the cavity also Dirichlet boundary conditions were applied, meaning that the excited species are de-excited at the wall (wall de-excitation). The diffusion model starts at $10 \mu \mathrm{s}$ to ensure that the electron temperature is near room temperature, which means that no radiating plasma species are created due to electron impact excitation. The initial condition of the diffusion model is a Gaussian with a FWHM equal to the FWHM at $10 \mu \mathrm{s}(5 \mathrm{~mm})$. The results of this model at $5 \mathrm{~Pa}$, $10 \mathrm{~Pa}$ and $15 \mathrm{~Pa}$ are plotted in figure 6 as well. The experimental data and the model show a reasonable agreement. On longer time scales $(>0.5 \mathrm{~ms})$, the experimental data and the model deviate slightly. The fact that the experimental results are slightly larger than the model could be caused by the fact that the model assumes a solid cavity wall, while in reality the cylindrical ring of the cavity is a mesh grid. The reason why the experimental results are sometimes smaller than the model is yet unknown. Since the experiments and the model are in reasonable agreement, the expansion is governed by diffusion of radiating plasma species at time scales longer than $10 \mu \mathrm{s}$.

In conclusion, the expansion on time scales longer than $10 \mu \mathrm{s}$ is driven by diffusion and subsequent wall de-excitation. The experimental results show a reasonable agreement with a simplified model describing the expansion of the radiating plasma species density distribution.

\section{The influence of the pressure on the radiating plasma species density}

In this section, the influence of the pressure on the radiating plasma species density is investigated. First, the maximum total emission intensity as a function of pressure is investigated. The processes responsible for the production of radiating plasma species are studied theoretically and the resulting theoretical pressure dependence is compared to the experimental results. Second, the decay of the total integrated

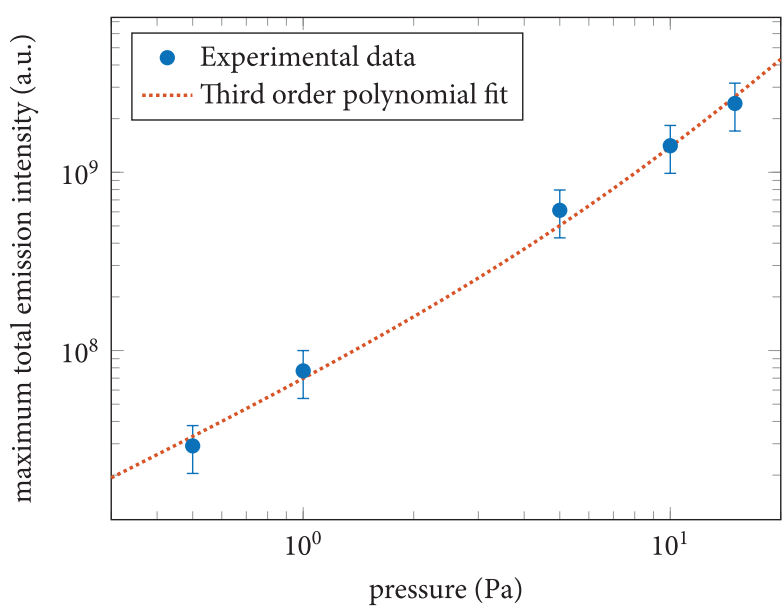

Figure 8. Maximum total emission intensity $I_{\max }$ as a function of argon pressure $p$. The experimental data is fitted with a cubic fit, where all coefficients should be larger or equal to zero. The fit yields the following expression: $I_{\max }(p)=(6 \pm 2) \times 10^{7} p+(1 \pm 1) \times 10^{7} p^{2}+(0 \pm 8) \times 10^{5} p^{3}$.

emission intensity on short time scales $(<10 \mu \mathrm{s})$ is studied. Finally, the decay of the total integrated emission intensity on long time scales $(>10 \mu \mathrm{s})$ is investigated and compared with a simplified diffusion model.

The maximum emission intensity, i.e. maximum density of radiating plasma species, reached directly after the EUV pulse is plotted in figure 8 as a function of gas pressure. The excited states can either be populated via photoionization and simultaneous excitation $[21,22]$ :

$$
\gamma+\mathrm{Ar} \longrightarrow \mathrm{e}^{-}+\mathrm{Ar}^{*^{*}}
$$

or by electron impact excitation [23]:

$$
\mathrm{e}^{-}+\mathrm{Ar} \longrightarrow \mathrm{e}^{-}+\mathrm{Ar}^{(+)^{*}},
$$

where $\mathrm{Ar}^{(+)^{*}}$ is either a radiating argon atom or ion. As discussed in the previous section, the most important loss term on this time scale is radiative decay:

$$
\operatorname{Ar}^{(+)^{*}} \longrightarrow \gamma+\operatorname{Ar}^{(+)}
$$

The density of radiating plasma species $n_{\mathrm{Ar}} *$ during irradiation with EUV photons is then given by:

$$
\frac{\mathrm{d}}{\mathrm{d} t} n_{\mathrm{Ar}} *=\frac{I_{0} n_{\mathrm{gas}} \sigma_{\mathrm{pe}}}{E_{\mathrm{ph}}}+k_{\mathrm{ee}} n_{e}(t) n_{\mathrm{gas}}-k_{r} n_{\mathrm{Ar}}{ }
$$

where $I_{0}$ is the EUV intensity, $n_{\text {gas }}$ the neutral gas density, $E_{\mathrm{ph}}$ is the photon energy, $\sigma_{\mathrm{pe}}$ is the cross section for photoionization and simultaneous excitation, $k_{\mathrm{ee}}$ is the electron impact excitation rate and $k_{r}$ is the natural decay rate of the radiating plasma species. In [10], it was shown that the electron density $n_{e}(t)$ depends quadratically on pressure:

$$
n_{e}(t)=\frac{N_{\mathrm{pe}} \sigma_{\mathrm{pi}} n_{\mathrm{gas}} I_{0} t}{E_{\mathrm{ph}}}\left(1+\frac{1}{2} \bar{k}_{\mathrm{ei}} n_{\mathrm{gas}} t\right),
$$

where $N_{\text {pe }}$ is the number of electron created per absorbed photon, $\sigma_{\mathrm{pi}}$ the photoionization cross section and $\bar{k}_{\mathrm{ei}}$ is the time average electron impact ionization rate. If this is substituted 
in equation (5), the production and loss of radiating plasma species yields:

$$
\begin{aligned}
\frac{\mathrm{d}}{\mathrm{d} t} n_{\mathrm{Ar}} *= & \frac{I_{0} n_{\mathrm{gas}} \sigma_{\mathrm{pe}}}{E_{\mathrm{ph}}} \\
& +k_{\mathrm{ee}} n_{\mathrm{gas}} \frac{N_{\mathrm{pe}} \sigma_{\mathrm{pi}} n_{\mathrm{gas}} I_{0} t}{E_{\mathrm{ph}}}\left(1+\frac{1}{2} k_{\mathrm{ei}} n_{\mathrm{gas}} t\right)-k_{r} n_{\mathrm{Ar}} *
\end{aligned}
$$

The solution of this equation at the end of the EUV pulse $\tau$ is:

$$
\begin{aligned}
n_{\mathrm{Ar}} *(\tau)= & \frac{I_{0}}{E_{\mathrm{ph}} k_{r}^{3}}\left[k_{r}^{2} \sigma_{\mathrm{pe}}\left(1-\mathrm{e}^{-k_{r} \tau}\right) n_{\mathrm{gas}}\right. \\
& +N_{\mathrm{pe}} k_{\mathrm{ee}} k_{r} \sigma_{\mathrm{pi}}\left(\mathrm{e}^{-k_{r} \tau}-1+k_{r} \tau\right) n_{\mathrm{gas}}^{2} \\
& \left.+N_{\mathrm{pe}} k_{\mathrm{ee}} k_{\mathrm{ei}} \sigma_{\mathrm{pi}}\left(-\mathrm{e}^{-k_{r} \tau}+1-k_{r} \tau+\frac{1}{2}\left(k_{r} \tau\right)^{2}\right) n_{\mathrm{gas}}^{3}\right] .
\end{aligned}
$$

This shows that the radiating plasma species density (and with it the emission intensity) depends cubically on the gas density, i.e. pressure, where all coefficients in this cubic expression are larger than or equal to zero. In this equation, the linear term is related to photoionization and simultaneous excitation, the quadratic term is related to electron impact excitation by electrons generated by photoionization and the cubic term is related to electron impact excitation by electrons generated by electron impact ionization. A third order polynomial is fitted through the experimental data points (figure 8). The cubic term in the fitted expression is, however, about zero $\left((0 \pm 8) \times 10^{5} \mathrm{~Pa}^{-3}\right)$, meaning that the emission intensity dependence on the pressure in the investigated pressure range can be approached quadratically. This also follows from theoretical considerations, if $k_{r} \approx 6 \times 10^{6} \mathrm{~s}^{-1}$ (see previous section), $k_{\mathrm{ei}} \approx 10^{-17} \mathrm{~m}^{3} \mathrm{~s}^{-1}$ (approximated from quadratic term in figure 6 and equation (13) in [10]) and $\tau \approx 10^{-7} \mathrm{~s}$ (duration of EUV pulse) is substituted in equation (8), it is found that the cubic term is about 3 orders of magnitude smaller than the quadratic term in the pressure range studied here $(<15 \mathrm{~Pa})$. This means that electron impact excitation by electrons generated by electron impact ionization is not significant in this pressure range. From this, it is concluded that the radiating plasma species are mainly generated via I) photoionization and simultaneous excitation and II) electron impact excitation by electrons originating from photoionization.

As discussed in section 3, the emission intensity, i.e. the number of radiating species, decreases rapidly during the first $10 \mu \mathrm{s}$. It was concluded that the time scale of this rapid decrease was determined by the natural lifetime of the excited states. Hence, the initial decay in emission intensity should not depend on the pressure. This can be observed in the experiments shown in figure 9 as well. This figure shows the normalized emission intensity during the first $5 \mu$ s for various argon pressures. It appears that the emission intensity decreases a little slower at pressures of $0.5 \mathrm{~Pa}$ and $1 \mathrm{~Pa}$ than at pressures of $5 \mathrm{~Pa}, 10 \mathrm{~Pa}$ and $15 \mathrm{~Pa}$ on time scales longer than $1 \mu \mathrm{s}$, while on time scales shorter than $1 \mu$ s the decay appears to be similar. To quantify this, the decay is fitted on two time intervals, i.e. between

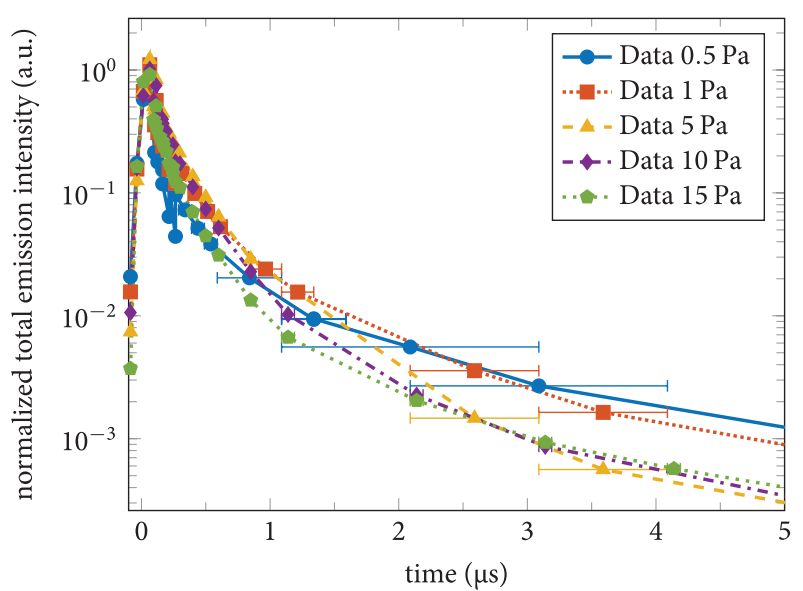

Figure 9. Normalized total emission intensity of the EUV-induced plasma before $5 \mu$ s at various pressures. The horizontal error bars represent the gate width of the iCCD camera.

100-300 ns and between 2-8 $\mu$ s, with an exponential function. In both of these time intervals, the resulting decay rates do not vary with pressure within the margin of error and are $(5.8 \pm 0.4) \times 10^{6} \mathrm{~s}^{-1}$ and $(4.7 \pm 0.4) \times 10^{5} \mathrm{~s}^{-1}$ respectively.

There is a difference in the decay between $5 \mathrm{~Pa}, 10 \mathrm{~Pa}$ and $15 \mathrm{~Pa}$ on time scales longer than $10 \mu$ s (figure 10). This means that the natural lifetime, which is independent of pressure, does not dominate the decay time on these time scales. Quenching neither influences the decay, since the typical time scale for quenching is longer than $0.3 \mathrm{~s}$. It follows that, the decay time should be dominated by diffusion and subsequent wall de-excitation. To verify this, the decay of the total emission intensity, i.e. number of radiating plasma species, on time scales longer than $10 \mu$ s is modelled with the simplified diffusion model discussed in the previous section. The results from the model are plotted in figure 10 as well. To compare the total emission intensity of the experiments and of the models, the modelled intensity is normalized to the experimental intensity. The experimental data and the model are in reasonable agreement, demonstrating that diffusion and subsequent wall de-excitation govern the decay of the total emission intensity on time scales longer than $10 \mu \mathrm{s}$. Naturally, also radiative decay occurs on these time scales, however, the decay rate is slower than the time scales associated with diffusion.

In conclusion, the radiating plasma species density depends quadratically on the pressure in the pressure range studied here $(<15 \mathrm{~Pa})$. The radiating plasma species are produced by photoionization and simultaneous excitation and by electron impact excitation by electrons generated by photoionization. At short time scales $(<10 \mu \mathrm{s})$, the decay of radiating plasma species does not depend on pressure, since it is governed by radiative decay. On longer time scales ( $>10 \mu \mathrm{s})$, the decay of radiating plasma species is dominated by diffusion and subsequent wall de-excitation.

\section{Comparison of the expansion of the electron and radiating plasma species density distribution}

In [12], the expansion of the electron density distribution was studied. In this section, this expansion will be compared to the 


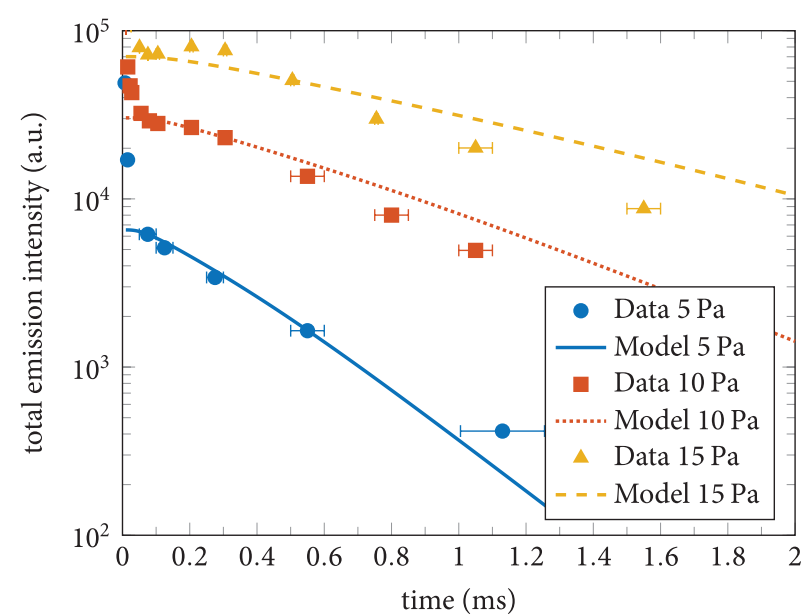

Figure 10. Total emission intensity of the EUV-induced plasma at various pressures. The horizontal error bars represent the gate width of the iCCD camera.

expansion of the radiating plasma species density distribution as discussed in section 4 .

The FWHM of the electron density distribution and the emission intensity profile, i.e. radiating plasma species density distribution as shown in figure 6, is plotted in figure 11. Since the shapes of the radial profile of the electron density distribution and emission intensity are slightly different, the absolute FWHMs are hard to compare in detail. However, it gives a rather intuitive insight to compare the time scales of the expansion.

In [12], it was shown that the expansion of the electron density distribution is dominated by ambipolar diffusion, meaning that the expansion of the ion density distribution should be comparable to the expansion of the electron density distribution on reasonably long time scales $(>1 \mu \mathrm{s})$. Since the expansion of the electron density distribution, i.e. the ion distribution, is much faster than the expansion of the radiating plasma species density distribution observed here (see figure 11), most likely ions are not the main source of emission. This means that the emission intensity at time scales longer than $10 \mu \mathrm{s}$ is dominated by light emitted by radiating neutral argon atoms, which is in contrast with the situation at time scales $<10 \mu$ s where the ions were found to be the main radiators of visible light (see section 4 ).

\section{The influence of the cavity walls on the plasma dynamics}

In previous sections it was concluded that at time scales shorter than $10 \mu \mathrm{s}$ the decay of the total integrated emission intensity is dominated by radiative decay, while at time scales longer than $10 \mu \mathrm{s}$ the decay is dominated by diffusion and subsequent wall de-excitation. This means that if the cavity is removed, the decay before $10 \mu$ s should not be influenced, while the decay after $10 \mu$ s should be slower since in this case radiative losses influence the decay rate and not wall de-excitation. Moreover, the cavity wall should limit the expansion of the radiating plasma species density distribution to the size of the cavity. To verify this, the cavity is removed to study its influence on the decay dynamics.

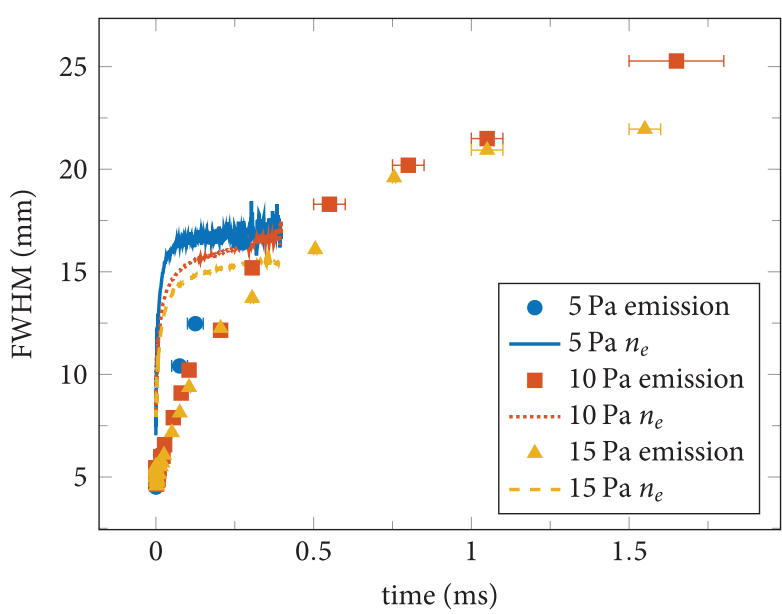

Figure 11. FWHM of the electron density distribution and spatial emission profile as a function of time for various pressures. The horizontal error bars represent the gate width of the iCCD camera.

The influence of the cavity walls is clearly visible in figure 12, which shows the total emission intensity as a function of time with and without cavity. It should be noted that the total emission intensity is a measure for the total number of radiating plasma species and not the density of radiating plasma species. The total emission intensity with cavity is about a factor 2 lower than the total emission intensity without cavity. This difference is explained by the transmission of the wall of the cavity, which is about $50 \%$ (see section 2). As expected, there is no difference between the decay before $10 \mu \mathrm{s}$ with or without cavity. The decay after $10 \mu \mathrm{s}$ is, as expected, significantly slower without cavity. As already mentioned, this is caused by the absence of wall de-excitation in the case without cavity.

It should be noted that without cavity, wall de-excitation will also occur when the radiating plasma species have reached the wall of the vacuum vessel. However, since no wall effects are observed on the time scales studied here, the radiating plasma species most likely have not reached the wall yet.

The FWHM of the radiating plasma species density distribution at a pressure of $5 \mathrm{~Pa}$ as a function of time with and without cavity is plotted in figure 13. It should be noted that by removing the cavity, the wall of the cavity is basically replaced by the wall of the vacuum vessel, which is about $8 \mathrm{~cm}$ from the plasma centre. In the beginning (until about $50 \mu \mathrm{s}$ ), the FWHM is similar with and without cavity. Hence, as expected, the expansion is not influenced by the cavity wall. When the radiating plasma species reach the wall of the cavity, the wall limits further expansion and the FWHM reaches an asymptote, while without cavity, the radiating plasma species density distribution continues to expand. Eventually, in the case without cavity, the wall of the vessel will limit further expansion, however, this is not observed on the time scales studied here.

The total emission intensity without cavity deviates from that with cavity after $10 \mu \mathrm{s}$, while the FWHM deviates after about $50 \mu \mathrm{s}$. This can be explained as follows. The lids of the cavity are closed to the plasma (about $10 \mathrm{~mm}$ ) than the cylindrical side-wall (about $33 \mathrm{~mm}$ ). The total emission intensity 


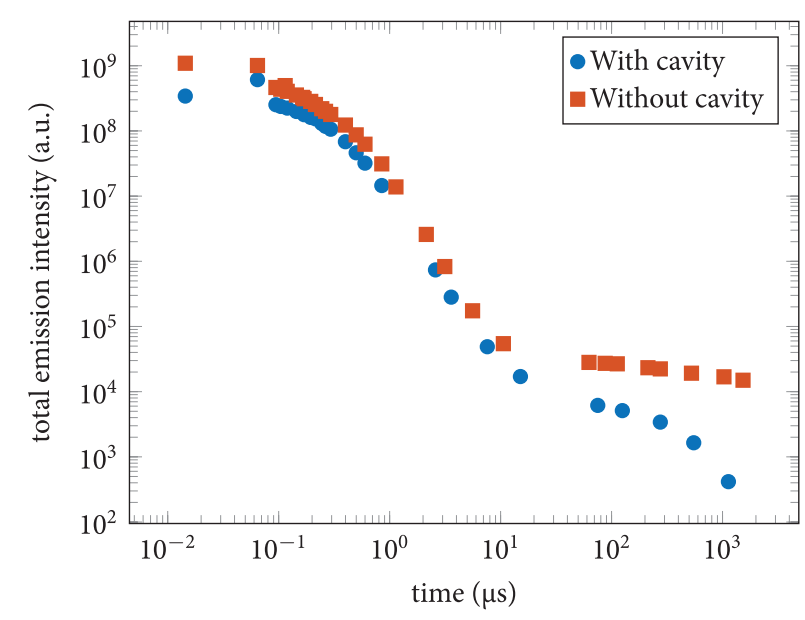

Figure 12. Total emission intensity as a function of time with and without cavity.

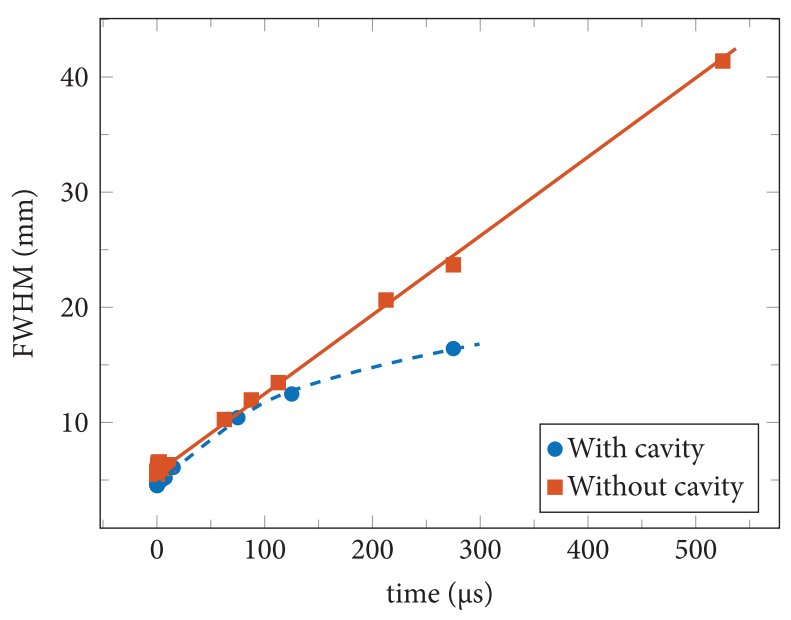

Figure 13. Full width at half maximum (FWHM) of the emission intensity as a function of time with and without cavity. The lines are a guide to the eyes.

will already start to deviate when the radiating particles reach the lids of the cavity, while the FWHM is not influenced until the radiating particles reach the cylindrical side-wall.

In conclusion, in this section the influence of the cavity walls on the radiating plasma species dynamics was studied. The wall of the cavity limits further expansion of the radiating plasma species density distribution and enhances the decay rate of the total emission intensity due to de-excitation at the wall.

\section{Conclusions}

The optical emission from a pulsed EUV-induced plasma in argon was studied.

It was found that the emission intensity, i.e. density of radiating plasma species, depends quadratically on the pressure in the pressure range studied here $(<15 \mathrm{~Pa})$. The linear term of the quadratic dependence is due to photoionization and simultaneous excitation by EUV photons, while the quadratic term is due to electron impact excitation by electrons generated by photoionization.
The decay of radiating plasma species can be divided into two phases. At time scales shorter than $10 \mu$ s (first phase), the decay is dominated by radiative decay of radiating plasma species. During this first phase, the emission intensity rapidly drops several orders of magnitude, while the radiating plasma species density distribution hardly expands. As a result, the plasma emission observed by eye appears to be confined along the EUV beam. At longer time scales (second phase, $>10 \mu \mathrm{s}$ ), the decay is governed by diffusion and subsequent de-excitation at the wall. The experimental decay and expansion during this phase correspond well with the theoretical results obtained from a simplified diffusion model. Since emission is visible in the entire cavity volume during this second phase, also radiative decay occurs, however, this decay rate is slower than the time scales associated with diffusion.

It was found from the experiments that the electron density distribution, and extrapolated the ion density distribution, expands much faster than the radiating plasma species density distribution. From this difference in the expansion speed it can be concluded that the emission intensity on longer time scales $(>10 \mu \mathrm{s})$ mainly originates from radiating neutral argon atoms.

The wall of the cavity influences the dynamics of the radiating plasma species due to wall de-excitation. As a result, the expansion is limited by the walls of the cavity. It should be noted that without cavity, the expansion is limited as well, but by the wall of the vacuum vessel. Furthermore, the decay rate of the total number of radiating plasma species increases due to de-excitation at the walls of the cavity.

\section{Acknowledgments}

The authors would like to acknowledge ASML for their financial support and the opportunity to use its EUV sources.

\section{References}

[1] Bartnik A, Fedosejevs R, Wachulak P, Fiedorowicz H, Serbanescu C, Saiz E, Riley D, Toleikis S and Neely D 2013 Laser Part. Beams 31 195-201 (www.journals. cambridge.org/abstract_S0263034613000050)

[2] Bartnik A, Wachulak P, Fiedorowicz H, Jarocki R, Kostecki J and Szczurek M 2013 Radiat. Phys. Chem. 93 9-13

[3] Bartnik A, Fiedorowicz H and Wachulak P 2014 Phys. Plasmas 21073303

[4] Bartnik A, Wachulak P, Fiedorowicz H, Fok T, Jarocki R and Szczurek M 2014 Phys. Scr. T 161014061

[5] OSullivan G et al 2015 Phys. Scr. 90054002

[6] van der Velden M, Brok W, van der Mullen J, Goedheer W and Banine V 2006 Phys. Rev. E 73036406

[7] van der Velden M 2008 Phd Thesis Eindhoven University of Technology Eindhoven (http://alexandria.tue.nl/ extra2/200810574.pdf)

[8] Dolgov A, Yakushev O, Abrikosov A, Snegirev E, Krivtsun V M, Lee C J and Bijkerk F 2015 Plasma Sources Sci. Technol. 24035003

[9] van der Horst R M, Beckers J, Nijdam S and Kroesen G M W 2014 J. Phys. D. Appl. Phys. 47302001 
[10] van der Horst R M, Beckers J, Osorio E A and Banine V Y 2015 J. Phys. D. Appl. Phys. 48285203

[11] van der Horst R M et al 2015 J. Phys. D. Appl. Phys. submitted

[12] van der Horst R M, Beckers J, Osorio E A and Banine V 2015 J. Phys. D. Appl. Phys. 48432001

[13] Bergmann K, Schriever G, Rosier O, Müller M, Neff W and Lebert R 1999 Appl. Opt. 385413

[14] Bergmann K, Rosier O, Lebert R, Neff W and Poprawe R 2001 Microelectron. Eng. 57-8 71-77

[15] Kalal M and Nugent K A 1988 Appl. Opt. 27 1956-9

[16] Bogaerts A and Gijbels R 2002 Spectrochim. Acta B 57 1071-99
[17] Kramida A, Ralchenko Y, Reader J and NIST ASD Team 2013 NIST Atomic Spectra Database (ver. 5.1) http://physics.nist. gov/asd

[18] Beckers J, van der Horst R, Osorio E and Banine V 2015 Plasma Sources Sci. Technol. submitted

[19] Banine V 2014 EUV Lithography: Historical perspective and road ahead Inaugural lecture Eindhoven Universiteit of Technology Eindhoven http://repository.tue.nl/778657

[20] Read P and Meyer M P 2000 Restoration of Motion Picture Film (Oxford: Butterworth-Heinemann) p 24

[21] Wijesundera W and Kelly H P 1987 Phys. Rev. A 36 4539-42

[22] Wijesundera W and Kelly H 1989 Phys. Rev. A 39 634-43

[23] Phelps A V and Petrovic Z L 1999 Plasma Sources Sci. Technol. 8 R21-44 